\title{
ROBUST SPARSE REPRESENTATION FOR ADAPTIVE SENSING OF TURBULENT PHENOMENA
}

\author{
Ion Candel $^{1,2}$, Cornel Ioana ${ }^{1}$, Bertrand Reeb $^{2}$ \\ ${ }^{1}$ GIPSA-lab, Grenoble Institute of Technology, 38402 Saint Martin d'Heres, France \\ E-mail: \{Ion.Candel, Cornel.Ioana $\}$ gipsa-lab.grenoble-inp.fr \\ ${ }^{2}$ Electricite de France, Division Technique Generale, 38040, Grenoble, France \\ E-mail : \{Ion.Candel, Bertrand.Reeb $\} @$ edf.fr
}

\begin{abstract}
In this paper, we propose a method for turbulence characterization using sparse representation of channel's impulse response. We consider the case of moving vortices created naturally or artificially that do not conserve their physical properties when observed at two distinct positions in space. Existing amplitude-based techniques fail to provide an accurate representation when the physical properties of the dynamic turbulence are altered. A two stages approach is proposed in this paper. The first one deals with the design of robust waveforms for sensing of turbulent phenomena. The second stage consists in sparsely representing the decomposition of the turbulence's impulse response, based on a physically driven decomposition basis. Tests carried out in a reduced scale experimental facility show, on real data, the efficiency of the turbulence tracking. We compare several types of signals and show that wide band signals are best suited for the application, achieving a high resolution combined to excellent results in terms of robustness.
\end{abstract}

Keywords — sparse representation, wide band waveforms, underwater acoustic propagation, turbulence, decomposition.

\section{INTRODUCTION}

The dynamics of aquatic phenomena can be understood as the propagation of vortices produced either naturally (natural turbulence embedded in water flow, marine life such as fish and mammals) or artificially (underwater obstacles, submarines, vessels). These vortices are detected and analyzed using pairs of acoustic transducers. If a vortex intersects the 
path between two pairs of active transducers, the signatures observed on the received signal's amplitudes are quantifiable. However, specific phenomena associated to the context (attenuation, dispersion, obstacles, etc.) alter the signature when the turbulence travels to another pair of transducers and thus the similarity between the two signatures can be weak.

An alternative is to decompose the impulse responses corresponding to the two transducers pairs on basis of elementary functions. In order to adapt the basis to the context of our application, two concepts are studied in this paper. First, we propose to design a decomposition basis inspired by the physical characteristics of the vortex "seen' by the impulse response of two propagating acoustic paths.

The second concept is the one of the sparse representation [1]: the analyzed phenomena can be recovered only using some of the decomposition coefficients that are relevant with respect of the minimization criterion, thus obtaining a sparse representation of the phenomena. At this level, we show the interest of the $L_{l}$ norm.

The article is structured as follows: section 2 presents an analysis of the best waveform to carry turbulence information, section 3 presents the theoretical concepts used in our work: waveform decomposition and sparse representation. Section 4 presents the main results and remarks of our work and section 5 presents the concluding remarks and future work.

\section{TURBULENT FLOW INTERACTION WITH DIFFERENT WAVEFORMS}

The analysis of turbulent phenomena is of major importance in the field of hydraulic systems or monitoring of natural environments. Conventional techniques rely on short sine pulses that are transmitted into the turbulent environment. The turbulence modifies the amplitudes of pulses in a random way and the analysis of the amplitude modification, when analyzed in bi-static configurations, conducts to the estimation of the turbulence's dynamics [2]. However, these techniques quickly reach their limitations because they provide little information regarding the dynamics of the turbulent flow. It is therefore necessary to construct more advanced waveforms to better understand the evolution of underwater turbulence.

The first research direction consists of adaptive waveform design that will be used for robust sensing of the turbulence. As a reference, typical to existing techniques, the short pulses are used as emitted signal. Two types of signals are then investigated for their potential to deliver a most accurate description of the turbulence dynamics: frequency shift keying (F.S.K.) signals and linear frequency modulations (CHIRPS). These signals are illustrated in the figure 1. 

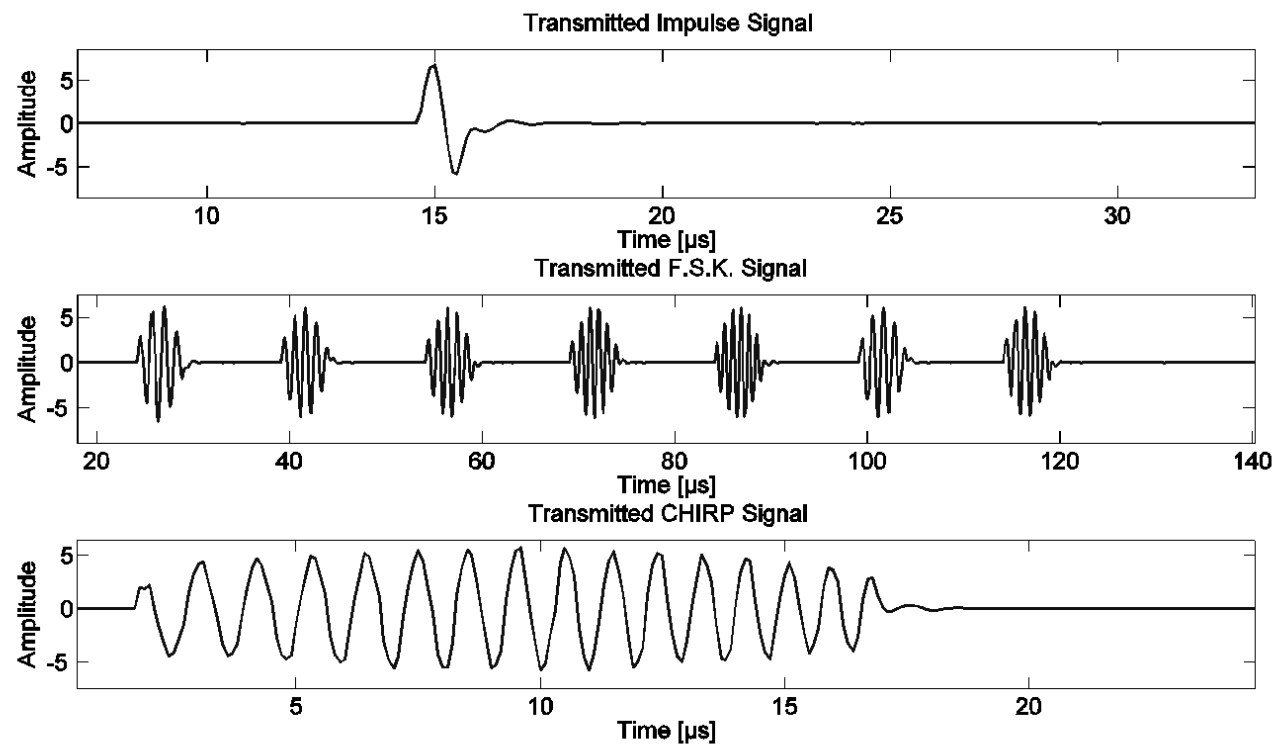

Figure 1. Different types of signals used to characterize turbulent phenomena.

In order to illustrate the motivation of our approach, the next example shows the evolution of impulse responses obtained via a matched filtering procedure, corresponding to the three types of signals, when the sensing waveforms interact with a moving vortex.

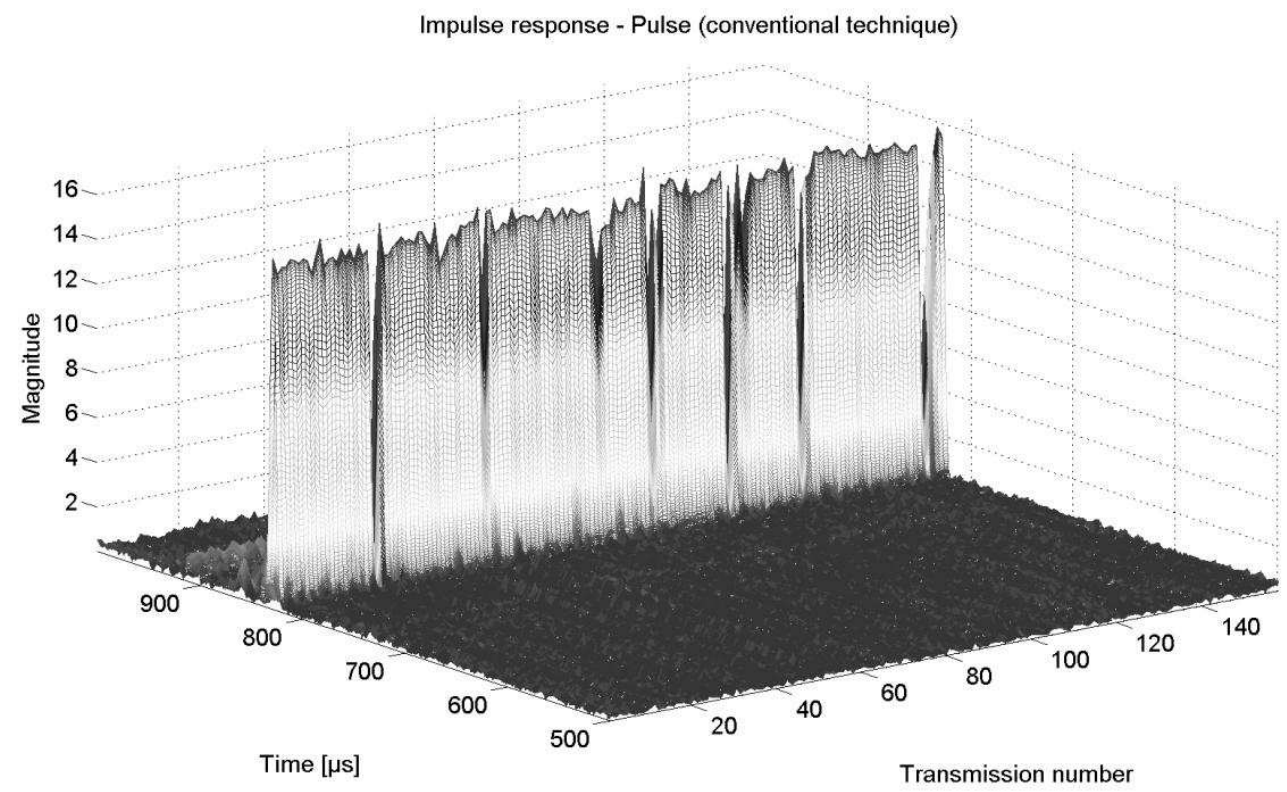

Figure 2. Impulse responses - pulse (conventional techniques). 


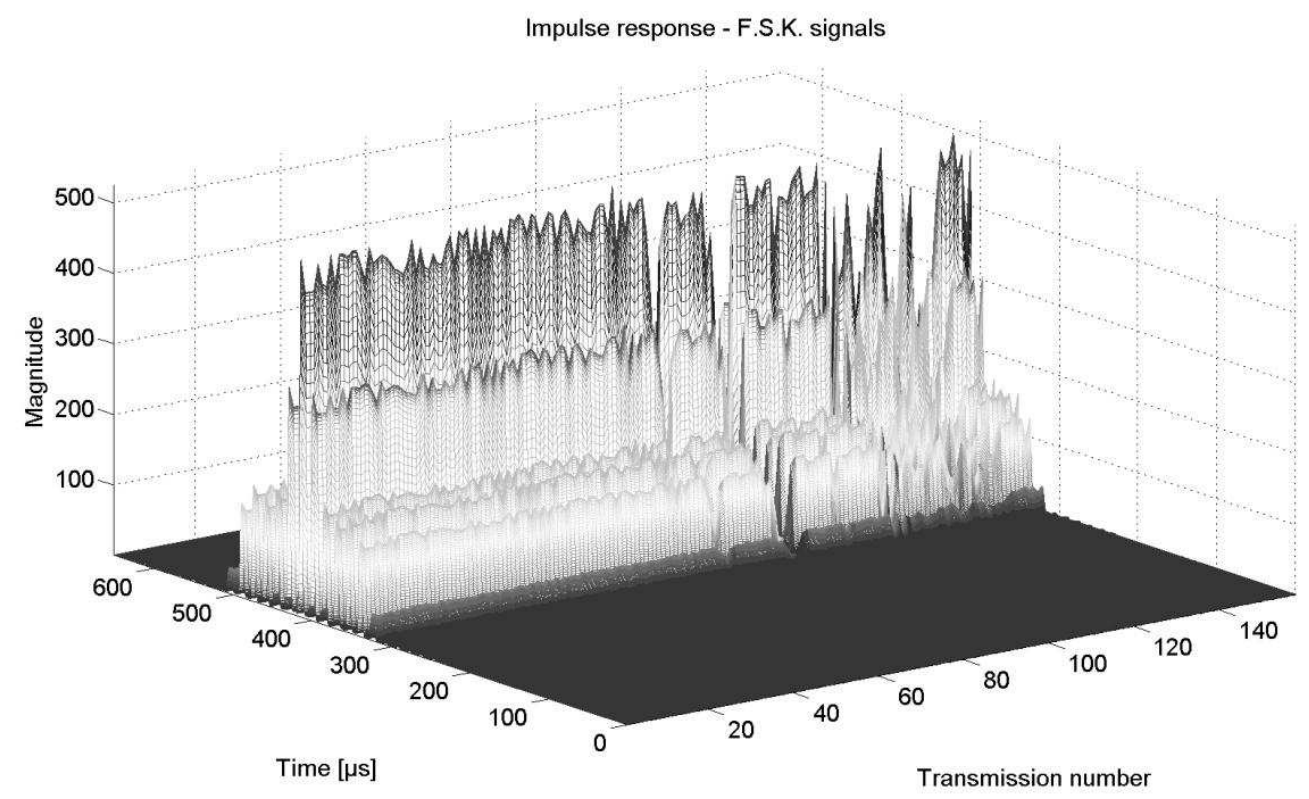

Figure 3. Impulse responses - FSK signals.

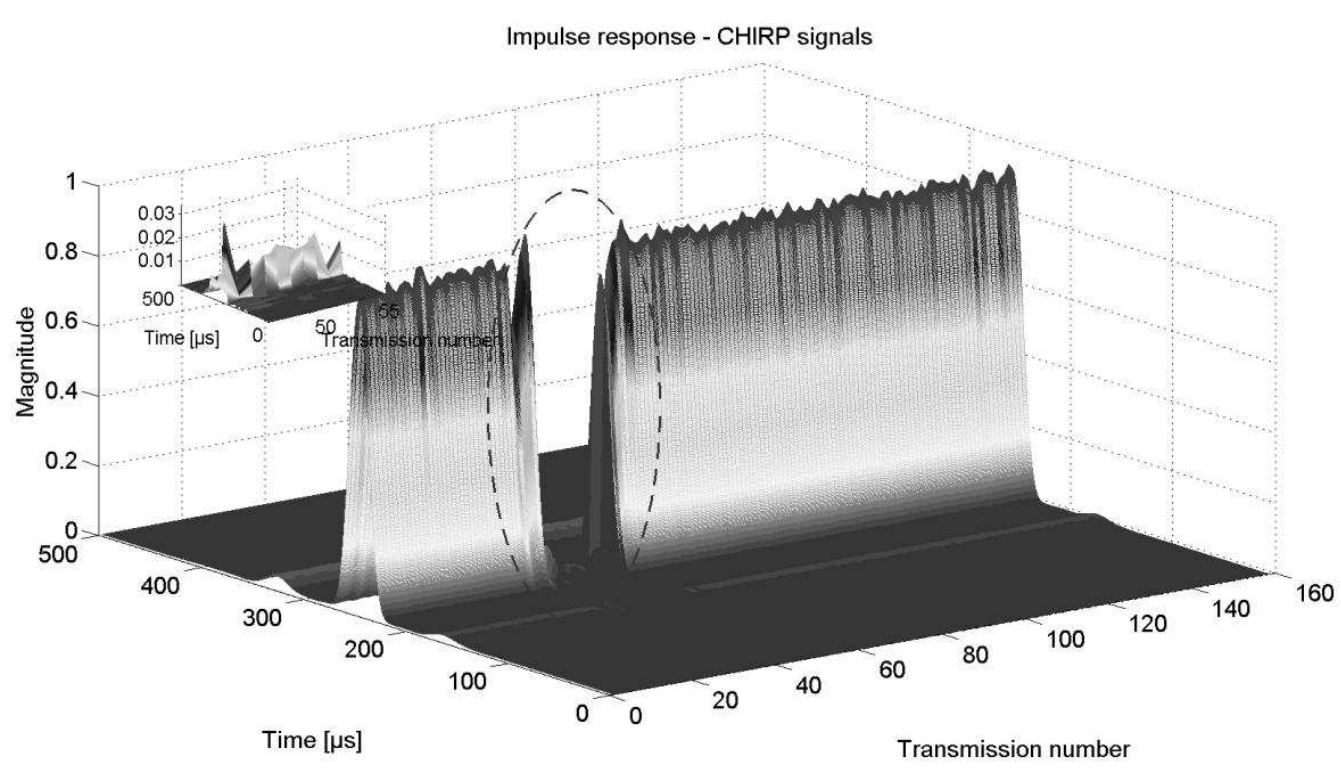

Figure 4. Impulse responses - CHIRP signals.

Figure 2 shows that due to the short duration of the pulse and thus low level of transmitted energy, the turbulence signature is reduced and therefore this type of signal is not suited for our application. Little information can be extracted, as most methods rely on the time delay between the emitted and received signal and amplitude changes. In addition to that, the signals can be easily corrupted by unwanted interference (equipment noise, vibrations, echoes, etc.) and therefore the range of applications is limited. In figure 3 however, the results are improved, but a relevant signature is still unavailable. This is due 
to the short duration of the F.S.K. steps. Initially developed for coding binary information, the F.S.K. signals' capability of encoding turbulence information is limited. In addition, the on/off commutation of transducers in a short time (successive pulses that are injected into the transducers in short amount of time), such as the pulses in the first $120 \mu$ s in the second subplot of figure one can cause more problems. The acoustic transducers do not support very short energy keying times (of the order of tens of $\mu \mathrm{s}$ ) and received signals may be distorted. Also due to the short duration between these steps reflection might occur which render the technique less robust to interference.

Figure 4 illustrates the case of CHIRP signals. A more precise description of the turbulent phenomena is provided as expected. The reason is that the wide band signal's duration is large enough to capture the passage of a turbulent front even in situations where the transmission has a high attenuation (small detail in figure 4). This represents an increase in the sensitivity of the method.

Consider the emission of two wide band signals from figure 1 using two acoustic transducers, $s_{e l}(t)$ and $s_{e 2}(t)$, in the form of two linear frequency modulations, described by the following equation:

$$
s_{e 1}(t)=s_{e 2}(t)=A \cdot \exp \left[j \cdot\left(\omega_{0} \cdot t+\gamma \cdot \frac{t^{2}}{2}\right)\right],
$$

where $\omega_{0}+\gamma t$ is the linear frequency variation law. The received signals $s_{r 1}(t)$ and $s_{r 2}(t)$ for each transducer can be expressed, ignoring the noise, as:

$$
\begin{aligned}
& s_{r 1}(t)=A_{r 1}(t) \cdot s_{e 1}(t-\tau) \\
& s_{r 2}(t)=A_{r 2}(t) \cdot s_{e 2}(t-\tau)
\end{aligned}
$$

where $\tau$ is the propagation time of the vortex between the emission and reception transducers. The envelopes $A_{r 1}$ and $A_{r 2}$ in the equations (2.2) contain the information about the turbulence evolution. We calculate the impulse response of the system for each transmission by correlating the received signal with the emitted one [3]:

$$
\begin{aligned}
& h_{r 1}(t)=\int_{-\infty}^{\infty} A_{r 1}(t) \cdot s_{e l}(t-\tau) \cdot s_{e 1}(t) d \tau \\
& h_{r 2}(t)=\int_{-\infty}^{\infty} A_{r 2}(t) \cdot s_{e 2}(t-\tau) \cdot s_{e 2}(t) d \tau
\end{aligned}
$$


Based on (2.3), the turbulence signature can be perceived as an inhomogeneity that appears on an otherwise straight wave profile, as illustrated in figure 5 .

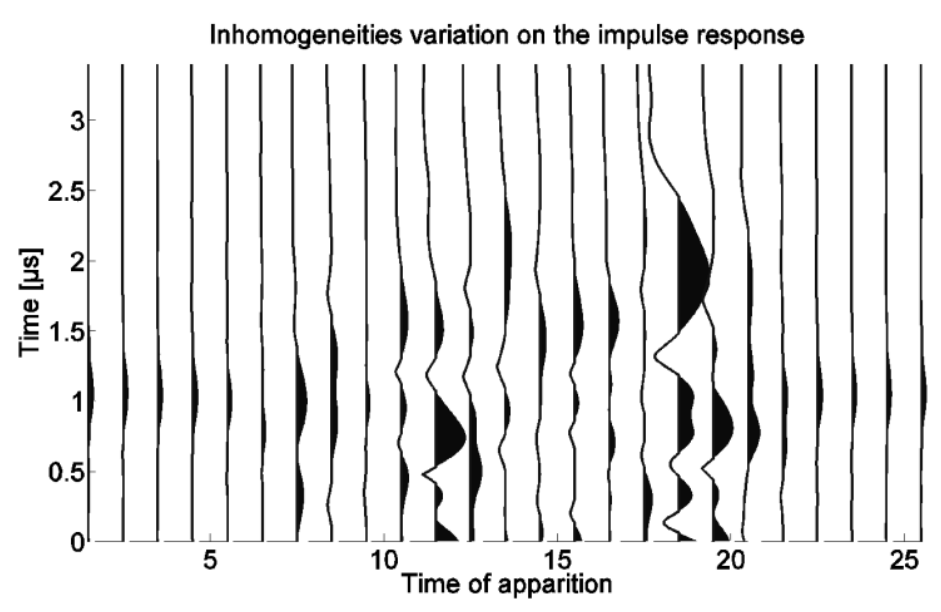

Figure 5. The signature of turbulence on the envelope of the impulse response.

\section{DECOMPOSITION BY SPARSE REPRESENTATION}

The general layout of the analyzed phenomena is illustrated in figure 6. Wide band signals are transmitted using two pairs of sensors placed on the Upstream and Downstream sides. The reason for a second pair of sensor resides in the fact that turbulence dynamics changes with distance. The sources of these changes must be identified (natural energetic decay, artificial and natural sources) or reduced in order to recover almost identical signatures of the same turbulence at two different paths separated by a certain distance.

In the absence of turbulence (such as a vortex), there are no changes in the shape of the impulse responses of the channels defined by the two pairs of transducers. The passage of turbulence between the transducers introduces modifications on the amplitudes of the impulse responses, calculated for each transmission, a fact that can be seen as signatures of the turbulence's source.

However, the vortex can change the characteristics while moving from one pair of sensors to the other, the similarity of the two signatures is low and can be considered as belonging to two different phenomena. The simple observation of the impulse responses is not enough for turbulence dynamics estimation. 
Therefore, the turbulence dynamics must be estimated by analyzing the impulse responses using their decomposition based on a physically - driven dictionary. That is, the decomposition attempts to highlight the common elements that will allow us to identify the turbulence.

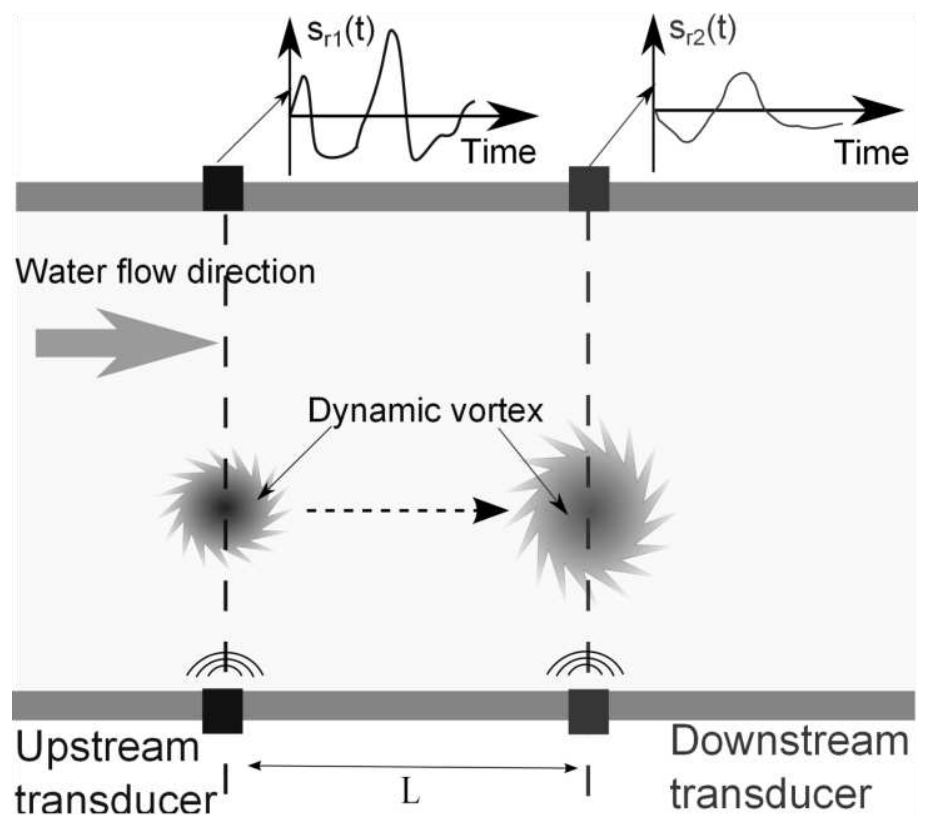

Figure 6. Vortex travelling with the flow of water

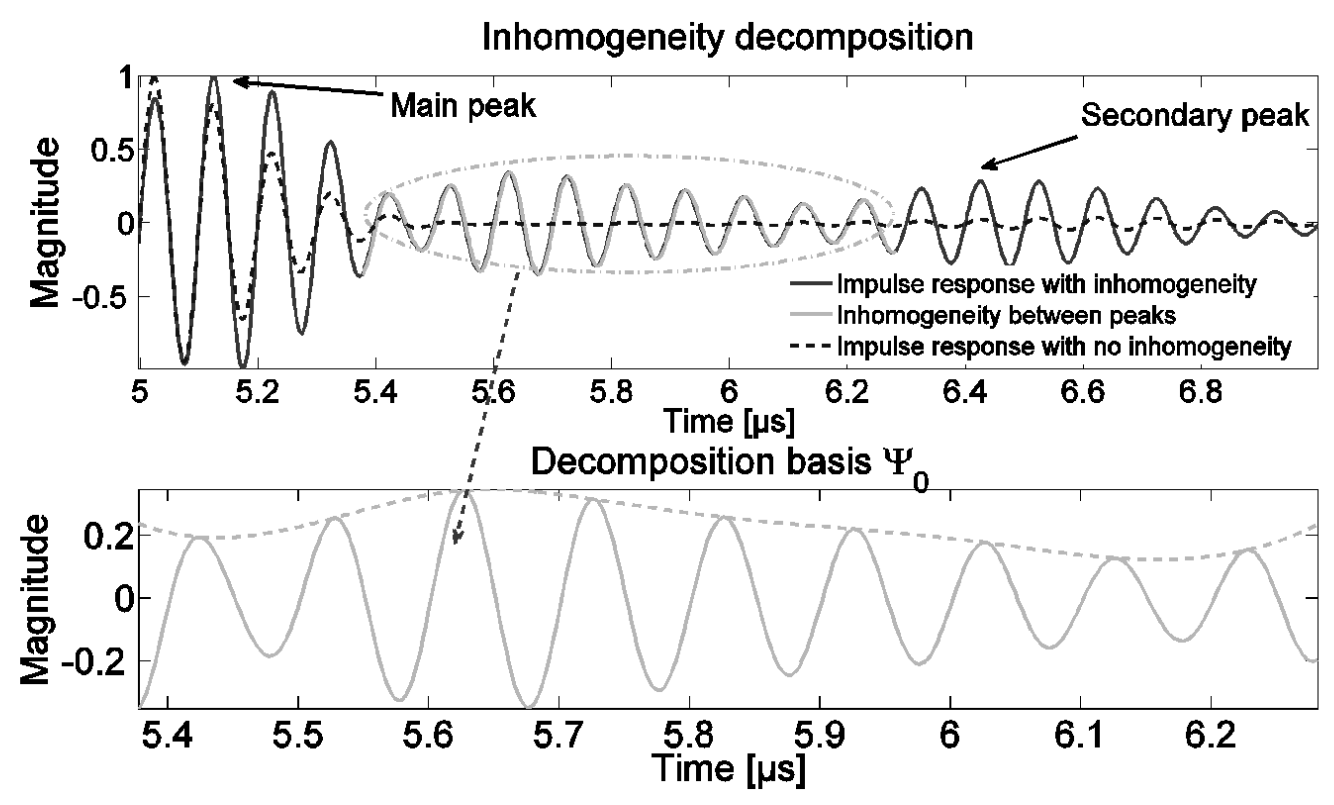

Figure 7. Decomposition principle of an turbulence extracted from an impulse response. 
However, the extraction of this signature must take into account the fact that due to occurring reflection of echoes, a secondary much smaller peak appears. This representation of the signature (amplitude data) cannot reveal accurate amount of information regarding the physical properties of the vortex. Therefore, we propose the analysis via a decomposition using an appropriate dictionary function constructed from the waveform received by the first pair of sensors (upstream acoustic path) having an amplitude variation similar to the one introduced by the vortex (see figure 7).

The basic principle of the decomposition is illustrated, using real data, in figure 7. Step one of the sparse representation consists in constructing the elements of the initial dictionary which is the same for both $h_{r l}(t)$ and $h_{r 2}(t)$, with a zero mean and a rapid decrease. The dictionary starts from an initial waveform $\psi_{0}$, which is a modulated sine wave centered on the resonance frequency of the transducers as illustrated in equation (2.4):

$$
\psi_{0}(t)=A(t) \cdot \exp \left(j \cdot \omega_{0} \cdot t\right)
$$

where $\omega_{0}$ corresponds to the resonance frequency of the transducers. The term $A(t)$ represents the amplitude modulation produced by the dynamic vortex over the impulse responses. The choice of this term is based on the physics of the vortex: from one path to another, the envelopes are similar, but due to the heterogeneity induced by the vortex the shape of the envelope suffers a contraction in time (figure 8).

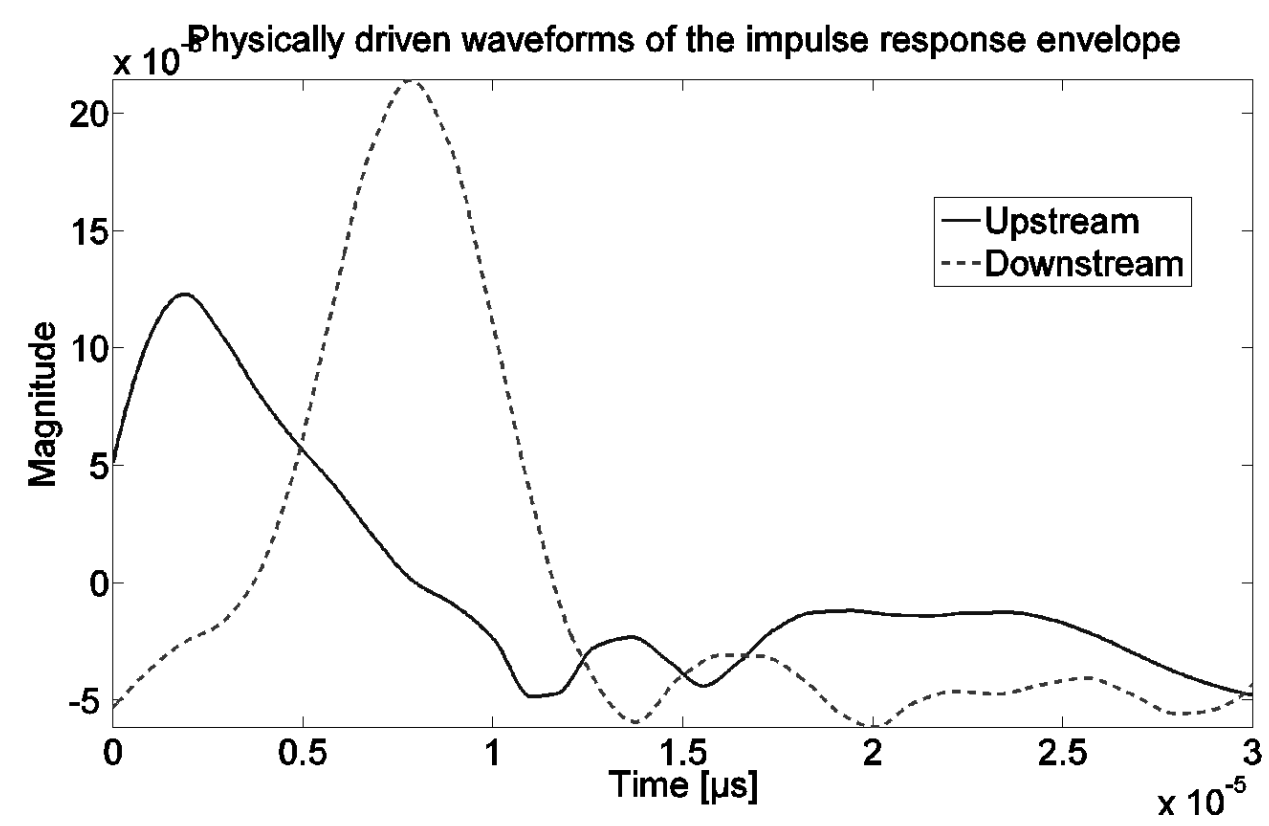

Figure 8 . The contraction of the impulse response envelope due to the dynamic vortex. 
Thus, the choice of $A(t)$ is made from the envelopes of the impulse responses that are affected the most by the turbulence, in order to provide a physically adapted dictionary for the decomposition. As the turbulence progresses through water, it keeps on enlarging due to its inertial character [4] and its energy decreases with distance.

Having defined the initial waveform we construct a set of functions that are shifted in time and scale, according to the continuous wavelet methodology [5], thus creating a dictionary of elementary functions, at different scales, shifted in time:

$$
\psi_{s, u}(t)=\frac{1}{\sqrt{s}} \cdot \psi_{0}\left(\frac{t-u}{s}\right), s=0.01 \cdot n ; n=1 \ldots N
$$

where $N$ is the maximum decomposition resolution, $s$ and $u$ are, respectively, the scale and shift parameters of the initial waveform $\psi_{0}$. The envelopes of the impulse responses from the Upstream side will be more contracted in time than the ones from the Downstream side. The reason for selecting the initial waveform among the impulse responses where the envelope's contraction is the highest resides in the decomposition process. The impulse responses will match the initial waveform $\psi_{0}$ at different scales highlighted by the $s$ parameter in (2.5). The representation that we wish to obtain for $h_{r l}(t)$ and $h_{r 2}(t)$ can be written, using the resulting coefficients, as follows [6] :

$$
\begin{aligned}
& h_{r 1}(t)=\sum_{i=1}^{N} C_{s, u}^{l} \cdot \psi_{s, u}(t) \\
& h_{r 2}(t)=\sum_{i=1}^{N} C_{s, u}^{2} \cdot \psi_{s, u}(t)
\end{aligned}
$$

where $C_{s, u}^{1}$ and $C_{s, u}^{2}$ are the $N$ resulting coefficients issued from the scalar product between the signal and each element of the $\psi_{s, u}(t)$ dictionary (basis) for the two impulse responses. The $C_{s, u}^{1}$ and $C_{s, u}^{2}$ coefficients represent the projection of the impulse function $h_{r}(t)$ on the $\psi_{s, u}(t)$ set of functions described in (2.4):

$$
C_{s, u}^{i}=<h_{r, i}, \psi_{s, u}>
$$

The second step of the sparse optimization consists in minimizing the number of coefficients obtained from the decomposition. This is defined as a problem of minimization [2] with solutions derived from convex optimization [7]. 
In the case of the turbulence estimation, a possible solution to the problem can be formulated as follows [8]: quantify the impact of each coefficient - basis pair between the two estimated turbulence signatures, in our context, and minimize the number of coefficients needed to construct two signatures $\tilde{h}_{r 1}$ and $\tilde{h}_{r 2}$ with minimum errors.

Based on (2.6), we calculate a residual for each one of the $N$ coefficients $\left(C^{i, j}{ }_{s, u}\right)$ and its corresponding dictionary element:

$$
R_{p}^{i}(t)=h_{r i}-\sum_{j=1}^{P} C_{s, u}^{i, j} \cdot \psi_{s, u}, P, j=1 \ldots N ; i=1,2
$$

where $R_{P}^{j}(t)$ is the residual calculated for the $P^{\text {th }}$ coefficients of the decomposition.

In order to quantify the impact of each residual with respect to the decomposition and minimize the number of coefficients, we can use the $L_{1}$ or $L_{2}$ norm for each of the $N$ residuals and select only the $\boldsymbol{K}$ coefficients corresponding to the number of coefficients for which the norm is minimum [9]. For each transmission, we obtain a residual matrix $R_{P}$ containing $N$ residuals calculated form (2.8). The optimum number of coefficients was fund by calculating a $L_{l}$ or $L_{2}$ norm for each line of the matrix (a residue):

$$
\begin{aligned}
& K=\min \left[\left\|\boldsymbol{R}_{P}^{i}(t)\right\|_{1}\right]-u \sin g \text { the } L_{1} \text { norm } \\
& K=\min \left[\left\|\boldsymbol{R}_{P}^{i}(t)\right\|_{2}\right]-u \sin g \text { the } L_{2} \text { norm }
\end{aligned}
$$

We choose to use the $L_{1}$ norm over the $L_{2}$ norm because the former leads to fewer coefficients than the latter as proved by the figure 9. In this figure, the plots for the $L_{1}$ and $L_{2}$ norms are zoomed in around their minima points.

The minimum of the $L_{l}$ norm correspond to the lowest number of coefficients used to represent sparsely the two signatures $\tilde{h}_{r 1}$ and $\tilde{h}_{r 2}$ :

$$
\begin{aligned}
& \tilde{h}_{r 1}(t)=\sum_{i=1}^{P} C_{s, u}^{l}(t) \cdot \psi_{s, u} \\
& \tilde{h}_{r 2}(t)=\sum_{i=1}^{P} C_{s, u}^{2}(t) \cdot \psi_{s, u}
\end{aligned}
$$

Since the basis of the decomposition is the same for both $\tilde{h}_{r 1}$ and $\tilde{h}_{r 2}(t)$, the two signatures will have a very strong likelihood, as it will be shown in the next sections. 


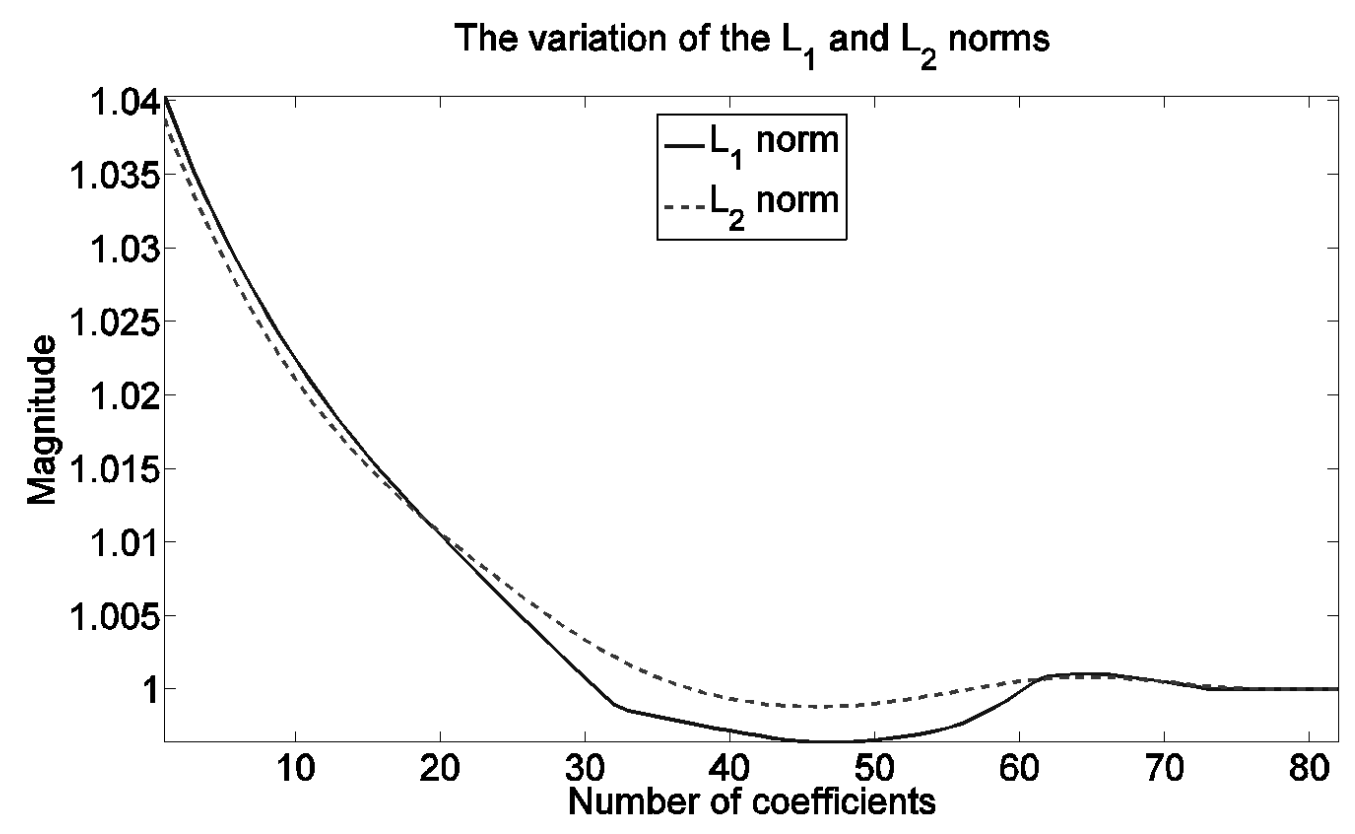

Figure 9. Variation of the $L_{1}$ and the $L_{2}$ norms.

\section{RESULTS}

The tests were carried out in our reduced scale experimental facility (figure 10). For our experiment, we used two pairs of $1 \mathrm{MHz}$ transducers placed on the outer walls of the tank. The length of the acoustic path was 1 meter at a depth of 40 $\mathrm{cm}$. Wide band signals were generated with a linear frequency modulation between $800 \mathrm{kHz}$ and $1.2 \mathrm{MHz}$, and were downloaded into a signal generator for transmission. The repetition rate of the signals was $1 \mathrm{msec}$ and the duration of the wide band signal is of $200 \mu$ s in order to avoid the overlap of echoes over the received signals. First, the pairs of transducers had to be set apart by a certain distance because of the occurrence of crosstalk caused by the wide beam angle of each transducer.

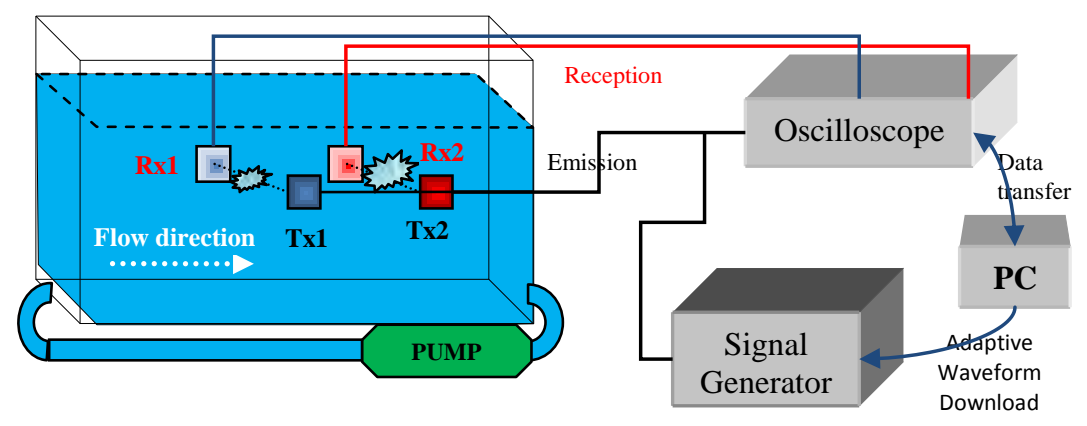

Figure 10. Reduced scale experiment test bench. 
Water flow was created using the recirculation pump in order to provide a background turbulence noise that would eventually superimpose over the simulated phenomena. Air was pumped through a tube submerged in the flow and the created turbulence intersected the two acoustic paths.

Figures 11 and 12 present the gradient of the impulse response for the Upstream and Downstream paths. Due to the use of wide band signals, the similarities between the two signatures are already visible.

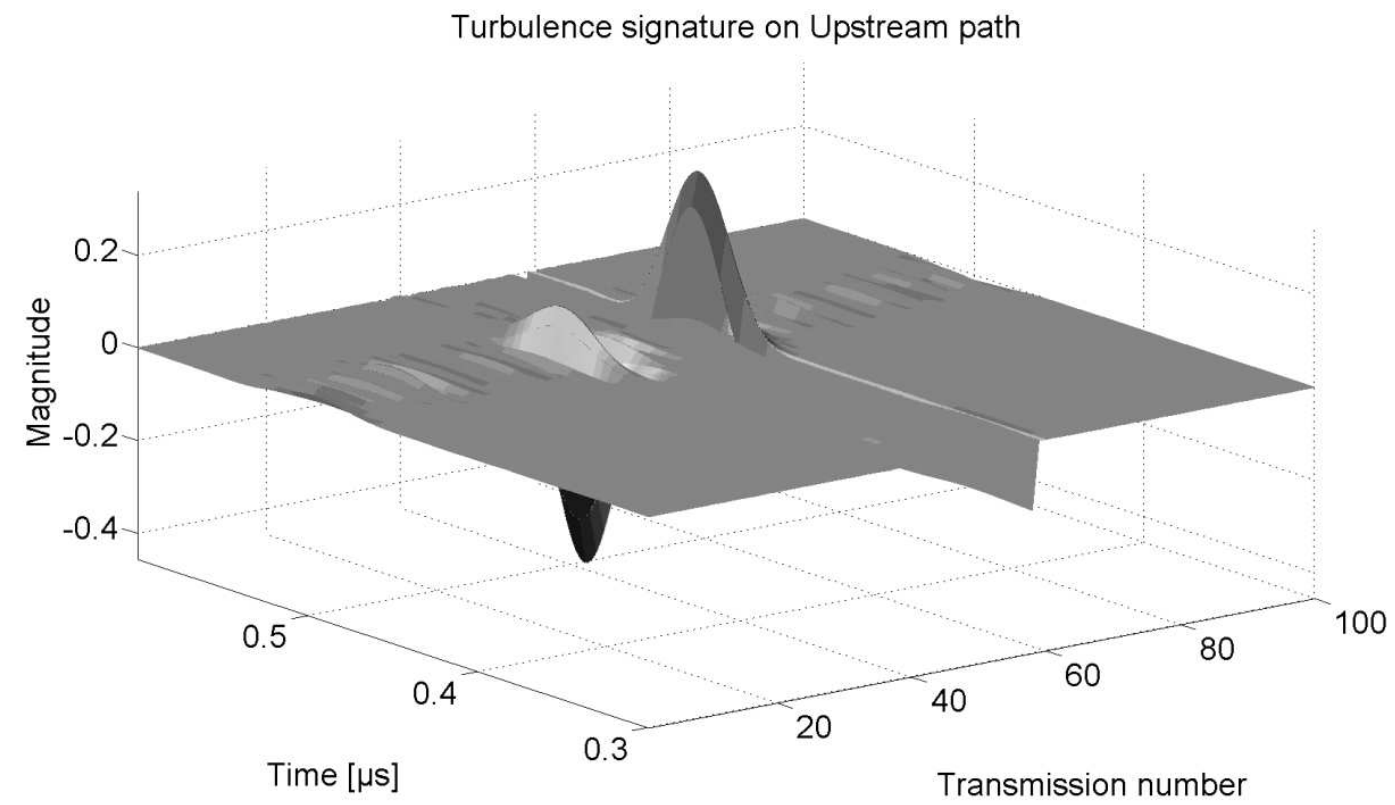

Figure 11. Turbulence signature for Upstream path.

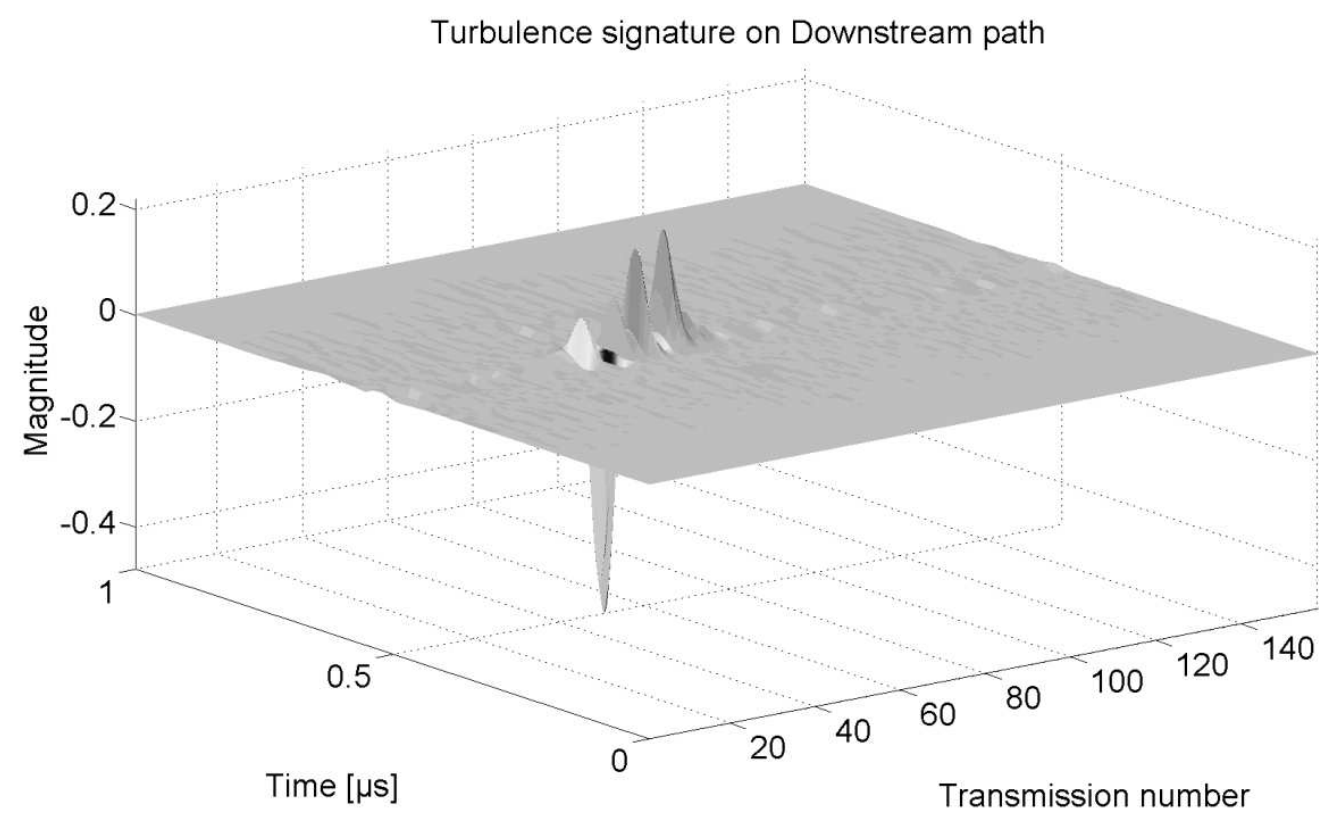

Figure 12. Turbulence signature for Downstream path. 
The scale factor of $s$ (decomposition basis parameter) was set to 0.01 due to resolution considerations (the number of elements in the dictionary) and the initial waveform, was selected among the zones extracted from the impulse response where the turbulence had the strongest effect, thus creating a physically driven basis. Each received signal yielded decomposition and a number of $K$ coefficients was calculated using the $L_{l}$ norm. It was no surprise that except for the moments where the effects of turbulence were strongest, the number of coefficients used for decomposition remained constant.

We compare our results in two ways: first, we show a significant improvement with a typical representation technique consisting in calculating the maxima values of the impulse response corresponding to each transmission as illustrated in figure 11. This technique is used extensively in certain flow metering applications presented in [10] and [11] and is sensible to interference induced by the measurement conditions.

Secondly, we use the results presented in figure 13 to calculate the average flow velocity between the acoustic paths. The accuracy of estimating the average velocity depends on the precision of estimating the time delay between the two acoustic paths. This is done by computing the cross correlation of the two signatures (Upstream and Downstream), as illustrated in figure 14 (a zoomed region displaying the main peaks of the two cross correlations).

In the second subplot of figure 13, we can observe an enhanced similarity between the signatures for the two acoustic paths, which describes an initial dynamic and random vortex. This result is possible only because of the physically driven dictionary that was used in the decomposition.

The second value (the real value calculated after the decomposition, highlighted on the red dotted trace with the vertical black line) corresponds to the correct orientation of the flow and a more pertinent $1.47 \mathrm{~m} / \mathrm{s}$ flow velocity is computed. Using the average flow velocities from multiple levels over a square section output the values of flow on that section. 

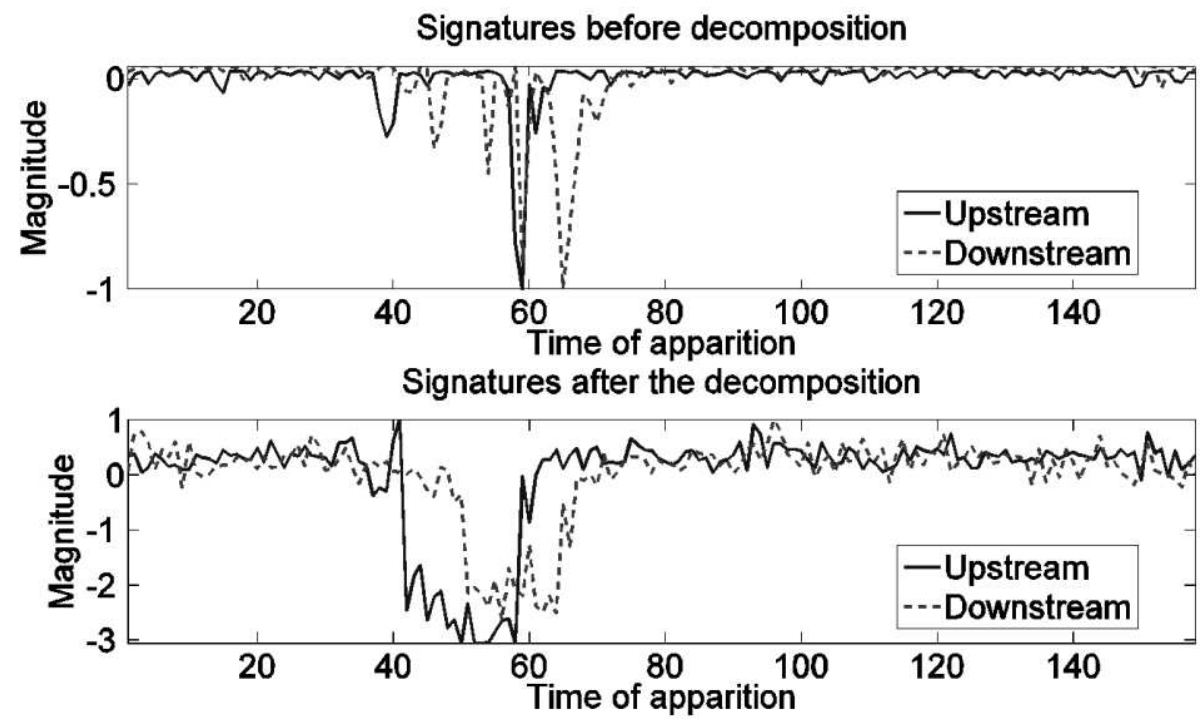

Figure 13. Turbulence representation before and after the sparse decomposition.

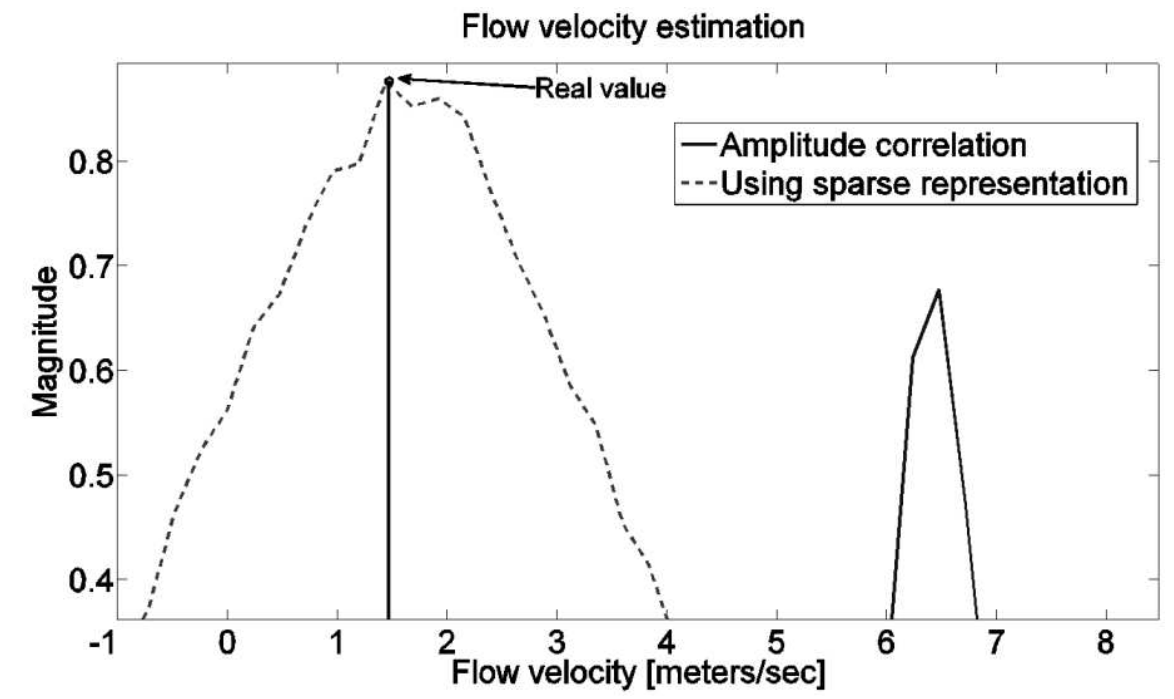

Figure 14. Flow velocity calculation using the cross correlation between the acoustic paths.

Accurate flow values calculated with existing methods are biased by unfavorable conditions: low turbulence levels [11] and transducer spacing [12]. Figure 14 shows that, by comparing with the initial estimation in figure 13, the sparse representation manages to provide a correct estimation of a turbulence translated in space. The purpose of sparse representation, as shown in [9], was to recover a signal using minimum number of coefficients from incomplete and altered measurements. 


\section{CONCLUSIONS}

In this paper, we show that sparse representation and adaptive waveform design techniques can be successfully used to recover a complete representation of turbulence from incomplete and contaminated measurements (sparse representation). We highlight the importance of adapting the content of the waveforms by studying the physics of the phenomena under measurement. This is due to the failure of a general accepted waveform to provide relevant and accurate information. Our method takes into account the turbulent nature of flow and the changes suffered by a waveform as it interacts with turbulence.

A small scale experiment consisted in generating turbulence was carried out and wide band signals were used to highlight the passage of the turbulence at two separated acoustic paths, all in the least favorable conditions.

Results obtained with our technique prove the theoretical concepts of sparse representation and adaptive waveform using large band signals: more information can be extracted, leading to more accurate results due to the robustness of the processing method.

Our work will focus in the future on combining adaptive waveform techniques with sparse representations aiming at finding a signal that is appropriate to underwater turbulence estimation using an orthonormal decomposition basis.

\section{REFERENCES}

[1] E. Candés, and T. Tao, "Decoding by Linear Programming", IEEE Transactions on Information Theory, IEEE Information Theory Society, vol.51, no. 12, pp. 4203-4215, December 2005.

[2] J. Lampa and D.D. Lemon, “Turbine Flow Measurement in Intakes with the Acoustic Scintillation Flow Meter”, Hydro Turbo 2012, 25 - 27 September 2012, Brno, Czech Republic.

[3] M. R. Bell, "Information Theory and Radar Waveform Design”, IEEE Transaction on Information Theory, vol. 39, No. 5, September 1993. 
[4] J. JIMENEZ, “The Contributions of A.N. Kolmogorov to the Theory of Turbulence”, Arbor CLXXVIII, 704, pp. 589 606, August 2004.

[5] M. Farge, "Wavelet Transforms and Their Applications to Turbulence”, Annual Reviewers Inc. Fluid Mechanics, No. 24, pp $395-457,1992$.

[6] Richard C. Baraniuk, Volkan Cevher, Marco F. Duarte, and Chinmay Hedge, "Model-Based Compressive Sensing", Cornell University Library, arXiv.com, 9 December 2009.

[7] S. Boyd, and L. Vandenberghe, Complex Optimization, Cambridge University Press, University Press, Cambridge, United Kingdom, 2004.

[8] S. S. Chen, D. Donoho, and M. Sanders, "Atomic Decomposition by Basis Pursuit", SIAM Review, Society for Industrial and Applied Mathematics, vol. 43, No. 1, pp. 129 -159, 2001.

[9] E. Candés, J. Romberg, and T. Tao "Stale Signal Recovery from Incomplete and Inaccurate Measurements", Communications on Pure and Applied Mathematics, California Institute of Technology, Pasadena, CA, Vol. 59, Issue 8, pp. $1207-1223$, August 2006.

[10] I. Candel, C. Ioana, D. Lemon, and B. Reeb, "Using Wide Band Signals for Obstacle Path Correction in Acoustic Scintillation Flow Meters", The $11^{\text {th }}$ International Conference on INFORMATION SCIENCE, SIGNAL PROCESSING and their Applications, ISSPA 2012, Montreal, Canada, July 3 - 52012.

[11] B. Reeb, I. Candel, G. Proulx, and D. Lemon, "Investigation of the Performance of Acoustic Scintillation Flow Meter when Turbulence Levels are Low”, Hydrovision International, Penwell, Lousiville, KY, July 21 - 272012.

[12] J.R. Marko, D.D. Lemon, "Negative Bias in ASFM Discharge Measurement in Short Intakes - Transducer Spacing", Proceedings IGHEM 2002, Toronto, Canada, 17 - 19 July 2002. 